\title{
Prediction of MIMO Channel Distribution with Transceiver Antenna Limitations
}

\author{
Srinivas , A. Leelavathi \\ M.Tech Student, Sr. Asst. prof, DIET
}

\begin{abstract}
The ability of ideal MIMO channels has a high-SNR slope that equals the minimum of the number of transceiver antennas. This work evaluates if this result holds when there are distortions from physical transceiver limitations. We prove analytically that such physical MIMO/SISO channels have a finite upper capacity limit, for any channel distribution and SNR. The high-SNR slope thus collapses to zero. This appears discouraging, but we prove the encouraging result that the relative capacity gain of employing MIMO/SISO is at least as large as with ideal transceivers. The entire results will be shown in MATLAB platform effectively.

Keywords: SNR, MIMO, Transceiver limitations, distortions
\end{abstract}

\section{Introduction}

Wireless communication enjoys considerable attention in the research community. Recent advances are mainly market driven by the demand for applications with increased data rates. Especially, wireless local area networks (WLANs), which aim at replacing wired computer network infrastructure with wireless communication technology, seem to raise a strong demand for further research and development. Different approaches to boost WLAN data rates have been considered in the past, as reflected in the amendments of the Institute of Electrical and Electronics Engineers (IEEE) 802.11 standard. First, data rates up to $11 \mathrm{Mbit} / \mathrm{s}$ are supported by IEEE $802.11 \mathrm{~b}$ compliant equipment. The modulation is direct sequence spread spectrum-based, which renders wireless channel equalization a complex task in the receiver. With the introduction of orthogonal frequency-division multiplexing techniques in the popular IEEE 802.11a and IEEE 802.11g standards, data rates up to $54 \mathrm{Mbits} / \mathrm{s}$ in a bandwidth of $20 \mathrm{MHz}$ can be realized with lowcomplexity channel equalization.

Channel bonding, i.e., expanding the bandwidth from $20 \mathrm{MHz}$ to $40 \mathrm{MHz}$, doubles the data throughput in some systems.

While higher throughput is anticipated, the available bandwidth for wireless systems is generally limited. This calls for technologies that achieve a higher throughput per bandwidth, i.e., higher spectral efficiency. Especially in crowded places, such as airports, train stations, or convention centers, low system capacity provided by today's technology poses a problem, which is the cause for insufficient data rates to individual users demanding basic data services. A candidate to achieve higher spectral efficiency in nextgeneration equipment is multiple-input multiple-output (MIMO) OFDM technology

\section{Mimo Concept}

MIMO technology allows multiple antennas at both transmitter and receiver to transmit independent data streams concurrently in the same frequency band. This principle, generally known as spatial multiplexing, results in a significantly higher spectral efficiency compared to single-input single-output (SISO) systems due to spatial diversity enabling a multiplexing gain. However, the detection algorithms employed in MIMO receivers have generally higher computational and hardware complexity than traditional SISO detection algorithms as demonstrated. While these results concern large network MIMO systems, there is another non-ideality that also affects performance and manifests itself for MIMO systems of any size: transceiver impairments. Physical radio-frequency (RF) transceivers suffer from amplifier non-linearities, IQ-imbalance, phase noise, quantization noise, carrier-frequency and sampling-rate jitter/offsets, etc. These impairments are conventionally overlooked in information theoretic studies, but this letter shows that they have a non-negligible and fundamental impact on the spectral efficiency in modern deployments with high SNR.

\section{Motivation for Work}

Motivated by the above discussion, we hereafter analytically assess the impact of residual RF impairments inthe transmitter and receiver hardware of MIMO systems. More specifically, we derive a new analytical expression for the MIMO ergodic capacity in independent and identically distributed. Rayleigh fading channels for arbitrary SNR values. Additionally, we also present asymptotic capacity expressions in the highSNR regime. In the low-SNR regime, we derive expressions for the minimum normalized energy per information bit required to convey any positive rate reliably and the wideband slope [13], which are the two key 
lowSNR parameters. This letter analyzes the generalized MIMO channel with transceiver impairments from [7]. We show that the capacity has a finite high-SNR limit for any channel distribution. The multiplexing gain is thus zero, which is fundamentally different from the ideal case in [1] (detailed above). The practical MIMO gain - the relative capacity increase over single-antenna channels - is however shown to be at least as large as with ideal transceiversThroughout our analysis, we find that the impact of residual impairments is marginal on low SNR systems, while it can substantially affect the performance of high SNR systems. In the last part, we analyze the ergodic capacity of large-dimensional MIMO systems with transceiver impairments and deduce asymptotic closed-form expressions for three typical cases. This provides valuable insights on how transceiver impairments affect large-scale (or "massive") MIMO systems.

\section{System Model}

Consider a flat-fading MIMO channel with $\mathrm{Nt}$ transmit antennas and $N_{r}$ receive antennas. The received signal is given as

$$
y=\sqrt{S N R} H x+n
$$

The intended signal $\mathrm{x}$ in (1) is only affected by a multiplicative channel transformation and additive thermal noise, thus ideal transceiver hardware is implicitly assumed. Physical transceivers suffer from a variety of impairments. The influence of impairments is reduced by compensation schemes, leaving a residual distortion with a variance that scales with SNR. A generalized MIMO channel is proposed in [6], [7] and verified by measurements. The combined (residual) influence of impairments in the transmitter hardware is modeled by the transmitter distortion

$$
y=\sqrt{\operatorname{SNR}} H\left(x+\eta_{t}\right)+n
$$

Note that $\eta \mathrm{t}$ is the mismatch between the intended signal $\mathrm{x}$ and the signal actually radiated by the transmitter; see Fig. 1. It is well-modeled as uncorrelated Gaussian noise as it is the aggregate residual of many impairments, whereof some are Gaussian and some behave as Gaussian when summed up

Fig 1 Block diagram of the MIMO channel with distortion noises from residual impairments in the transmitter and receiver hardware.

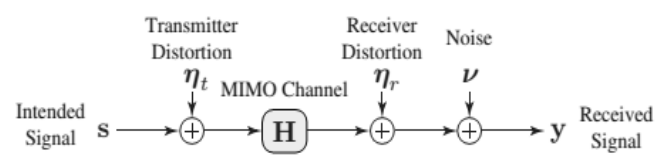

The distortion depends on the intended signal $\mathrm{x}$ in the sense that the variance vn(qn) is an increasing function of the signal power qn at the nth transmit antenna (i.e., the nth diagonal element of Q). We neglect any antenna cross-correlation in Yt. In multi-carrier (e.g., OFDM) scenarios, (2) can describe each individual subcarrier. However, there is some distortion leakage between subcarriers that makes qn less influential on $v_{\mathrm{n}}(\mathrm{qn})$. For simplicity, we model the leakage as proportional to the average signal power per antenna (i.e., the direct impact of what is done on individual antennas/subcarriers averages outwhen having many subcarriers). To capture a range of cases we propose

$$
v_{n}\left(q_{n}\right)=k^{2}\left((1-\alpha) q_{n}+\alpha \frac{\sum_{i=1}^{N_{t}} q_{i}}{N_{t}}\right)
$$

where the parameter $\alpha \in[0,1]$ enables transition from one $(\alpha=0)$ to many $(\alpha=1)$ subcarriers. The parameter $\kappa>$ 0 is the level of impairments. This model is a good characterization of phase noise and IQ-imbalance, while the impact of amplifier non-linearities grows non-linearly in SNR. We assume to operate in the dynamic range where the impact is almost linear.

\section{Channel Capacity}

In a fixed wireless environment, the fading coefficients vary slowly, so the transmitter can periodically send pilot signals to allow the receiver to estimate the coefficients accurately. In mobile environments, however, the fading coefficients can change quite rapidly and the estimation of channel parameters becomes difficult, particularly in a system with a large number of antenna elements. In this case, there may not be enough time to estimate the parameters accurately enough. Also, the time one spends in sending pilot signals is not negligible, and the tradeoff between sending more pilot signals to estimate the channel more accurately and using more time to communicate to get more data through becomes an important factor affecting performance. In such situations, one may also be interested in exploring schemes that do not need explicit estimates of the fading 
coefficients. It is therefore of interest to understand the fundamental limits of non-coherent multiple antenna communications.

$$
Y=\sqrt{\frac{S N R}{M}} H X+W
$$

with the only difference that $\mathrm{H}$ is not known at the receiver, as well as the transmitter. The channel capacity and error probability are defined similarly to their coherent counterparts. The computation is, however, usually much more complicated. The Shannon capacity of a time-invariant channel is defined as the maximum mutual information between the channel input and output. This is the maximum data rate that can be transmitted over the channel with arbitrarily small error probability. When the CSI is perfectly known at both the transmitter and the receiver, the transmitter can adapt its transmission strategy relative to the instantaneous channel state. If the channel is time variant, the ergodic capacity is the maximum mutual information averaged over all channel states. The ergodic capacity is typically achieved using an adaptive transmission policy where the power and data rate $\mathrm{v}$ ary relative to the channel state variations. In a multiple user scenario, MU MIMO allows the reuse of time and frequency resources. Due to the scattering in different scenarios, the users' wavefronts may have large.

\section{Outputs}

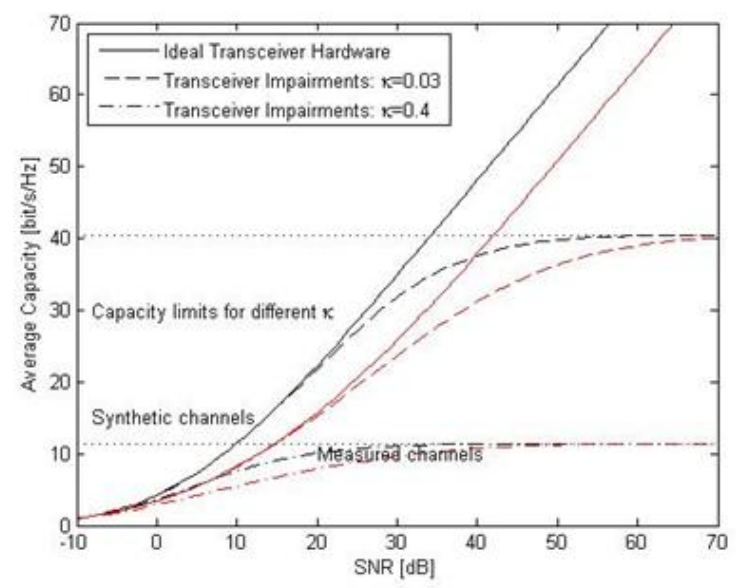

Fig 2 Average capacity of a $4 \times 4$ MIMO channel over different deterministic channel realizations, different levels of transceiver impairments, and $\alpha=1$ and $\mathrm{k}=0.03$ and $\mathrm{k}=0.4$

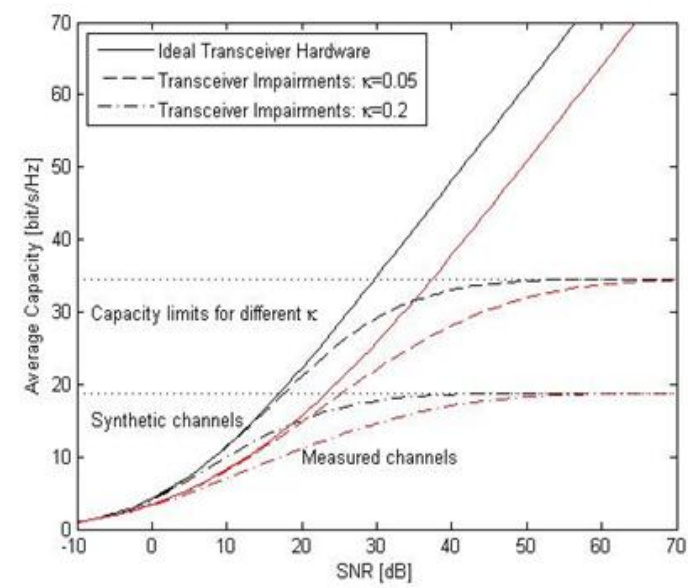

Fig 3 Average capacity of a $4 \times 4$ MIMO channel over different deterministic channel realizations, different levels of transceiver impairments, and $\alpha=1$ and $\mathrm{k}=0.05$ and $\mathrm{k}=0.2$ 


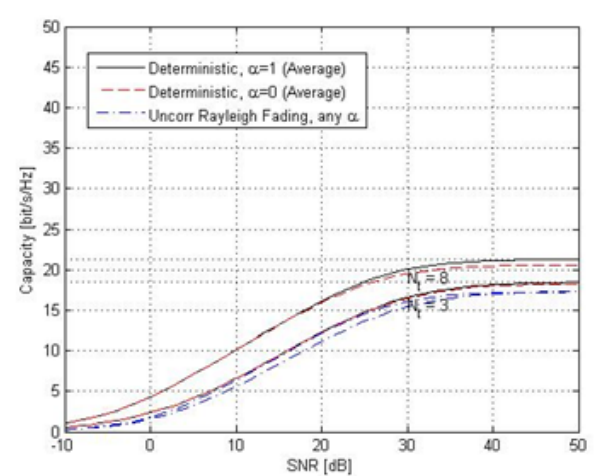

Fig 5 Capacity of a MIMO channel with $\mathrm{Nr}=2$ and $\mathrm{Nt}=8$ and 3 and impairments with $\kappa=0.05$. We consider different $\mathrm{Nt}$, channel distributions, and $\alpha$ values.

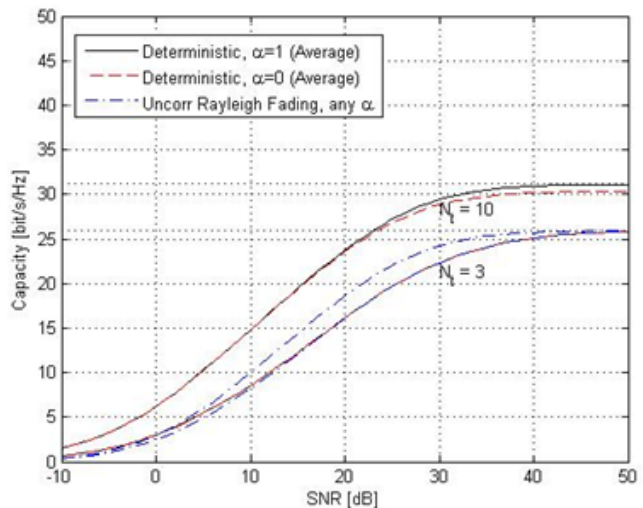

Fig 6 Capacity of a MIMO channel with $\mathrm{Nr}=3$ and $\mathrm{Nt}=10$ and 3 and impairments with $\kappa=0.05$. We consider different $\mathrm{Nt}$, channel distributions, and $\alpha-$ values.

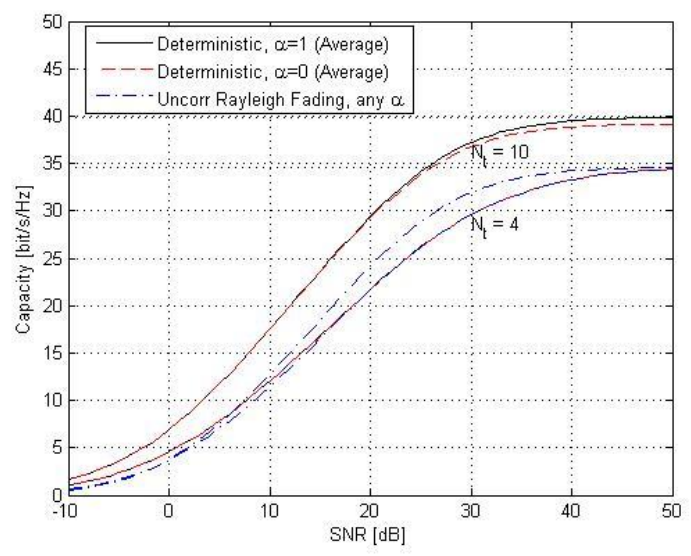

Fig 5 Capacity of a MIMO channel with $\mathrm{Nr}=4$ and $\mathrm{Nt}=10$ and 4 and impairments with $\kappa=0.05$. We consider different $\mathrm{Nt}$, channel distributions, and $\alpha$-values.

\section{Conclusion}

Residual RF hardware impairments can have a dramatic impact on the capacity of MIMO communication systems, especially on those operating at high SNRs (i.e., high-rate systems). In this paper, we analytically derived an ergodic capacity expression for a MIMO system with residual transceiver impairments, which applies for any finite number of antennas and the entire SNR range. This expression can be very easily evaluated, since it only contains elementary functions. This original result is explained by the distortion from transceiver impairments and that its power is comparative to the gesture power. The classic multiplexing gain is thus zero (see Eq. (7)). Nevertheless, the MIMO vigor grows roughly linearly through $\mathrm{M}=\min (\mathrm{Nt}, \mathrm{Nr}$ ) over the whole SNR range, thus showing the encouraging result that also physical system be capable of achieve great gains from employing MIMO and spatial multiplexing. scientific advance can reduce transceiver impairments, but in attendance is currently an opposite trend towards small low-cost low-power transceivers where the innate dirty RF effects are inevitable and the program is instead adapted to them. The point-to-point MIMO capacity limit in Theorem 1 is an upper bound for state of affairs amid extra constraints; for illustration, network MIMO, which is characterized by distributed power constraints and partial coordination both between transmit antennas and between receive antennas. The capacity in such scenarios consequently saturate in the high-SNR regimeeven in small networks where the analysis is not valid. Finally, note that the finite-SNR multiplexing gain decreases when adding extra constraints furthermore that impairments limit the asymptotic precision of channel acquisition schemes. 


\section{References}

[1] F. Rusek, D. Persson, B. Lau, E. G. Larsson, T. L. Marzetta, O. Edfors, and F. Tufvesson, "Scaling up MIMO: Opportunities and challenges with very large arrays,’IEEE Signal Process. Mag., vol. 30, no. 1, pp. 40-60, Jan. 2013.

[2] M. Kang and M.-S. Alouini, "Capacity of MIMO Rician channels," IEEE Trans. Wireless Commun., vol. 5, no. 1, pp. 112-122, Jan. 2006. [21] R. G. Bartle,The Elements of Integration and Lebesgue Measure. Wiley Online Library, 1995.

[3] A. Lozano, A. M. Tulino, and S. Verd' u, "Multiple-antenna capacity in the low-power regime," IEEE Trans. Inf. Theory, vol. 49, no. 10, pp. 2527-2544, Oct. 2003.

[4] Y. Zou, M. Valkama, and M. Renfors, "Digital compensation of I/Q imbalance effects in space-time coded transmit diversity systems," IEEE Trans. Signal Process., vol. 56, no. 6, pp. 2496-2508, June 2008

[5] M. Katz and S. Shamai, "On the capacity-achieving distribution of the discrete-time noncoherent and partially coherent AWGN channels,” IEEE Trans. Inf. Theory, vol. 50, no. 10, pp. 2257-2270, Oct. 2004.

[6] C. Studer, M. Wenk, and A. Burg, "MIMO transmission with residual transmit-RF impairments," in Proc. ITG/IEEE WSA, 2010.

[7] A. Lozano, R. Heath, and J. Andrews, "Fundamental limits of cooperation," IEEE Trans. Inf. Theory, submitted, arXiv:1204.0011.

[8] J. J. van de Beek, M. Sandell, and P. O. Börjesson, "ML estimation of time and frequency offset in OFDM systems,"IEEE Trans. Signal Processing, vol. 45, pp. 1800-1805, 1997

[9] I. E. Telatar, "Capacity of multi-antenna Gaussian channels," Bell Laboratories, Lucent Technologies,” Technical Memorandum, Oct. 1995, published in European Transactions on Telecommunications, Vol. 10, No. 6, pp. 585-595, Nov/Dec 1999.

[10] T.C.W.Schenk,M.M.deLaat,P.F.M.Smulders, and E.R.Fledderus, "Symbol timing for multiple antenna OFDM systems," in Proc. IEEE Vehicular Technology Fall 2006,vol.3, 2006, pp. 1521-1525.

[11] T. C. W. Schenk and A. van Zelst, "Frequency synchronization for MIMO-OFDM wireless LAN systems," inProc. IEEE Vehicular Technology Fall 2003., vol. 2, 2003, pp. 781-785.

[12] Per Zetterberg, Mats Bengtsson, and Bj"orn Ottersten, “Capacity Limits and Multiplexing Gains of MIMO Channels with Transceiver Impairments" 